\title{
Searching for Programme theories for a realist evaluation: a case study comparing an academic database search and a simple Google search
}

Susanne Coleman ${ }^{1 *}$ D, Judy M. Wright ${ }^{1}$, Jane Nixon ${ }^{1}$, Lisette Schoonhoven², Maureen Twiddy ${ }^{3}$ and Joanne Greenhalgh ${ }^{4}$

\begin{abstract}
Background: Realist methodologies are increasingly being used to evaluate complex interventions in health and social care. Programme theory (ideas and assumptions of how a particular intervention works) development is the first step in a realist evaluation or a realist synthesis, with literature reviews providing important evidence to support this. Deciding how to search for programme theories is challenging and there is limited guidance available. Using an example of identifying programme theories for a realist evaluation of Pressure Ulcer Risk Assessment Instruments in clinical practice, the authors explore and compare several different approaches to literature searching and highlight important methodological considerations for those embarking on a programme theory review.

Methods: We compared the performance of an academic database search with a simple Google search and developed an optimised search strategy for the identification primary references (i.e. documents providing the clearest examples of programme theories) associated with the use of Pressure Ulcer Risk Assessment Instruments (PU-RAIs). We identified the number of primary references and the total number of references retrieved per source. We then calculated the number needed to read (NNR) expressed as the total number of titles and abstracts screened to identify one relevant reference from each source.

Results: The academic database search (comprising CINAHL, The Cochrane Library, EMBASE, HMIC, Medline) identified 2/10 primary references with a NNR of 1395 . The Google search identified 7/10 primary references with a NNR of 10.1. The combined NNR was 286.3. The optimised search combining Google and CINAHL identified 10/10 primary references with a NNR of 40.2 .

* Correspondence: medscole@leeds.ac.uk

'Leeds Institute of Clinical Trials Research, University of Leeds, Leeds LS2 9JT,

UK

Full list of author information is available at the end of the article

C The Author(s). 2020 Open Access This article is licensed under a Creative Commons Attribution 4.0 International License, which permits use, sharing, adaptation, distribution and reproduction in any medium or format, as long as you give appropriate credit to the original author(s) and the source, provide a link to the Creative Commons licence, and indicate if changes were made. The images or other third party material in this article are included in the article's Creative Commons licence, unless indicated otherwise in a credit line to the material. If material is not included in the article's Creative Commons licence and your intended use is not permitted by statutory regulation or exceeds the permitted use, you will need to obtain permission directly from the copyright holder. To view a copy of this licence, visit http://creativecommons.org/licenses/by/4.0/. The Creative Commons Public Domain Dedication waiver (http://creativecommons.org/publicdomain/zero/1.0/) applies to the data made available in this article, unless otherwise stated in a credit line to the data. 


\begin{abstract}
(Continued from previous page)
Conclusion: The striking difference between the efficiency of the review's academic database and Google searches in finding relevant references prompted an in-depth comparison of the two types of search. The findings indicate the importance of including grey literature sources such as Google in this particular programme theory search, while acknowledging the need for transparency of methods. Further research is needed to facilitate improved guidance for programme theory searches to enhance practice in the realist field and to save researcher time and therefore resource.
\end{abstract}

Keywords: Realist evaluation, Programme theory, Scoping review, Literature searching, Information retrieval, Internet

\section{Background}

Realist methodology is increasingly used in the evaluation of complex health care interventions, to facilitate a deeper understanding of 'what works, how, for whom, in what circumstances and to what extent' [1-4]. The initial step of realist studies aims to identify programme theories underlying the intervention. A programme theory is an explanation of how and why an intervention is expected to work and is often expressed as a Context Mechanism Outcome (CMO) configuration. Programme theory identification is an iterative process and can involve a range of methods including reviewing existing literature, documentation review, interviews with stakeholders, focus groups and/or reviewing relevant theories from other literature $[2,3,5]$.

Ideas about programme theories can be found in a range of sources and are not limited to academic literature; they can be found in websites, blogs, newspaper articles, letters or even radio programmes [6]. Deciding how to search for programme theories is challenging as there is limited guidance for realist searches $[3,7,8]$ compared with systematic reviews [9-12]. While both searches employ a wide range of strategies, searches for programme theories focus on identifying a different type of source compared to systematic reviews. This stems from the different underlying aims of the reviews. Systematic reviews employing a conventional 'Cochrane style' methodology to answer questions of treatment/ diagnostic effectiveness, aim to identify all relevant empirical studies and often reflect strict inclusion/exclusion criteria e.g. for study design, outcomes, populations and settings. Multiple database searches and supplementary search techniques are recommended to reduce publication bias [11-14]. However, searches to support the identification of programme theories are concerned with identifying the full range of ideas and assumptions that underpin how the intervention is intended to work (rather than every paper containing a programme theory). These ideas often pertain to assertions about how the programme might work, the populations or situations in which the programme might work as intended and those where it might be expected not to work. Identifying these ideas often requires consideration of wider literature such as opinion papers, guidelines, blogs and organisation reports $[8,15]$ which may not be indexed in academic databases $[16,17]$. Furthermore, at this stage of any realist enquiry, the focus is on identifying plausible ideas, rather than evidence to underpin the validity of those ideas; the testing and refinement of programme theory (by juxataposing these ideas with evidence) occurs in the next stage of any realist enquiry. As such, the sources used to identify programme theory are not subject to specific study design considerations.

Designing a search with a high recall (sensitivity) and high precision is challenge for both a review of theories and systematic reviews. Searches are designed with the aim of finding all relevant papers, while minimising retrieval of irrelevant papers. At the same time the searches must be robust and transparent $[13,18]$. The most efficient way to achieve this for programme theory reviews remains unclear and many approaches are considered relevant, incorporating browsing library shelves, discussion with experts, citation tracking, web searching and cluster searching $[8,16,17,19-$ 21]. Google is considered a quick and easy way to search for grey literature and other sources of programme theories (eg websites, blogs, newspaper articles etc). However, it is not considered a first choice of resource for systematic searching due to the impact of personalised search history and lack of transparency over content [22].

The authors of the present paper became aware of these considerations while undertaking a scoping review to identify programme theories in preparation for a realist evaluation of the use of Pressure Ulcer Risk Assessment Instruments (PU-RAIs) by nurses in clinical practice. A realist evaluation is a theory driven evaluation concerned with facilitating a deeper understanding of 'what works, how, for whom, in what circumstances and to what extent' and involves identifying, testing and refining programme theories $[2,23]$. Initially the authors took a conventional empirical approach to literature reviewing and through this learning experience were able to highlight some of the problems encountered and how these were addressed (detailed in Table 1). Using this example we explore and compare several different 
(though not all) approaches to literature searching and highlight potential wider implications that could be relevant to others undertaking programme theory scoping reviews, as well as considerations for future apriori methodological studies of literature searching.

\section{Aim}

To compare the performance of a search of academic databases with a simple Google search and inform the development of an optimised search strategy for the identification of programme theories associated with the use of PU-RAIs.

\section{Objectives}

- Compare searches (academic database vs Google vs Google scholar) in identifying primary papers of the scoping review and the resulting screening task (number of references found) from each source.

- Identify reasons for non-retrieval of primary papers in existing searches.

- Test replicability of Google and Google Scholar searches.

- Develop improved search strategies to efficiently capture all primary papers.

\section{Method}

Firstly, we populated a 'Search Summary Table' [25] which listed the 10 primary papers and the sources we searched (CINAHL, The Cochrane Library, EMBASE, Google search engine, HMIC, Medline, SC's personal library) to record:

1) Where our searches had identified each reference in one or more of the sources

2) How many primary references were found per source

3) How many references were retrieved in total from each source

4) The number needed to read (NNR) for each source. NNR is a measure of the precision of a search [26] i.e. the number of titles and abstracts that are screened to identify one relevant reference.

5) In which sources the reference exists and therefore could be found by an 'optimum search'

The Search Summary Table captures information to determine the minimum set of sources needed to retrieve all primary references for both the academic database search, the Google search and for an 'optimum search', that is one which would identify all 10 primary papers. It allows a comparison between the performance of Google and academic database searches in terms of how many of the 10 primary references can be (and were) identified by each.
Table 1 Summary of methods and results of a Scoping Review of PU-RAl use in clinical practice

\section{Introduction}

An academic database search was undertaken to identify programme theories associated PU-RAI use. We were looking for a range of publication types and expected to find some grey literature in the academic databases due to their content coverage. This was supplemented with a Google search.

\section{Method}

A search strategy incorporating key words relating to risk assessment, pressure ulcers and other areas associated with risk assessment instruments including falls and frailty as well as theories and publication types (systematic review, commentary, opinion piece and editorial) (Appendix 1) was used. Five databases were searched from 1970 to May 2017 (Cochrane Database of Systematic Reviews, Ovid MEDLINE, Ovid HMIC, Ovid Embase, CINAHL EBSCOhost).

Inclusion criteria: Papers relating to RAls which describe, review, discuss, critique, provide a theoretical framework or provide stakeholder accounts of use.

Exclusion criteria: Papers relating to RAls as a research tool or to assess psychometric properties.

Screening: After removal of duplicates, the titles, abstracts, and key words of identified papers were screened for relevance by the primary reviewer (SC). Papers considered potentially relevant were obtained in full and reviewed to assess validity and similarity (repetition) of identified programme theories. A 10\% random sample of those screened and those potentially relevant were independently screened by a second reviewer (JG). Following identification of papers including relevant theories, the primary papers i.e. those providing clear detail of the possible context and mechanisms through which the intervention/ programme is intended to work, were included in the review.

An example of a clearly articulated programme theory associated with this review is detailed below: 'staff should receive adequate training to ensure they are competent to use the pressure ulcer risk assessment tool. This is especially relevant for novice practitioners who do not have the extensive clinical judgement skills that experienced practitioners have. Therefore risk assessment tools may be especially beneficial for them, particularly when admitting patients in order to prioritise care' [30]. To summarise one theory that can be drawn from this is that that novice practitioners who do not have extensive clinical judgement skills (Context) may particularly benefit from PU-RAI use (Mechanism) to help them prioritise care (Outcome).

Results

Despite the database search identifying 2790 potentially relevant papers (after removal of duplicates), only 2 were considered primary papers (Fig. 1).

Subsequent Google Search

Due to the inadequate results of the academic database search a simple Google search of 'pressure ulcer risk assessment' and 'pressure ulcer risk assessment tools' was undertaken in September 2017. As a large volume of papers were identified and limited resources were available the screening was limited to the first five pages of the Google search. This identified 71 potentially relevant papers (after removal of duplicates) with 6 additional primary papers being of relevance. Two further papers were identified from the researchers' knowledge (Fig. 1).

Secondly, we identified and evaluated instances where references were not found in academic database/Google searches despite their being available in the respective search engine. Understanding the reasons for nonretrieval would help design of new search strategies with improved retrieval of primary papers. We checked terms used for Pressure Ulcers, Risk Assessment Tools and Theories/Systematic review study types within our primary studies to see if we had not used these terms in our original search strategies. The database fields 
searched (such as title, abstract, index word) was important to determine if we had used the correct terms but in the wrong fields.

Next, we developed and tested improved search strategies for Google and the academic databases to capture all primary papers while retrieving a minimum number of records. We developed an optimised search to identify search methods that could improve precision without losing sensitivity for programme theory reviews. To do this we incorporated our learning of which terms were used in the primary papers and the fields in which they appear in the database record. All search terms used in the original searches were checked to identify if any were redundant (i.e. did not retrieve primary papers). We tested if these search terms could be removed from the search strategy or searched in a more precise manner, e.g. only searching for terms within the title field of database records. We used the NNR calculation to compare the precision i.e. the proportion of results retrieved by the search that are relevant [27] across different sources. We also tested the Google search strategy in Google Scholar to test the impact of searching Google rather than Google Scholar in the original search. Since some literature reviews search Google Scholar rather than Google, a comparison of two could inform the development of the 'optimum search'.

Finally, in order to understand the replicability of Google and Google Scholar for this particular search, we ran two identical searches on Google and Google Scholar using two PCs based in in the same building, on the same day within a $2 \mathrm{~h}$ period. Neither researcher had logged into a Google account on this PC, reducing expected personalisation of results. The first search used the terms 'Pressure UIcer Risk Assessment' and the second search used the terms 'Pressure Ulcer Risk Assessment Tools'. The first five pages of Google and Google Scholar results were downloaded for each search. The replicability test compared results on the first five pages of downloaded records since this was the number downloaded in the original Google search.

\section{Results}

The academic database searches identified 2790 references (after duplicate removal), and the first five pages of the Google search identified 71 references (after duplicate removal). The search results were supplemented with 2 known relevant references from SC's personal library that had not been identified by either the academic or Google searches. The (NNR) to identify a primary paper from the combined academic databases, Google search and SC's personal library was 286.3 (2790 refs + 71 refs +2 refs / 10 primary refs).

\section{Database comparison}

Figure 2 shows the databases where primary papers were found in the searches. It also shows the databases that held these references and could have been located with 'the optimum search' but were not necessarily identified with our search. Seven of the ten included references were identified in the Google searches [24, 28-33]. One of these [34] was also found in the database search. Therefore, six references were uniquely found via the Google searches. The NNR for the Google searches was 10.1 references.

Across the academic databases the CINAHL search identified two primary papers. Several database searches were 'redundant'; HMIC did not identify any relevant references, and The Cochrane Library, EMBASE and Medline searches found two duplicates [34, 35] also found in the CINAHL search. A further two primary papers were identified in SC's personal library [36, 37]. Using our search strategies, the 10 primary papers could have been identified using three sources; CINAHL, Google and SC's Personal Library, rather than the seven sources (Fig. 3). The NNR for the academic searches was 1395 while for the combined academic databases and Google search it was 286.3 .

\section{Reasons for non-retrieval in existing searches}

Figure 1 shows that some primary paper references were available in CINAHL, EMBASE, Medline and Google, but were not identified by the original searches. Table 2 lists the reasons for non-retrieval. All 10 primary references were available in Google, however only 7 appeared in the first five pages of search results from the original two Google searches.

When the original Google searches were replicated in 2019 the 10 primary references were found within the first 12 pages of Google (i.e. the first 216 records if it is assumed there are 9 references per page on average in Google search results). This indicates that if a larger set of results (216) had been screened from the original search, all 10 primary papers could have been identified with this one source. However, this is an estimate, as we do not have the data for a download of the first 12 pages from the search in 2017.

Five references were not indexed in the academic databases we searched (Table 2). These included a guideline [37], blog item [30], conference abstract [31], book chapter [33] and one journal article [36]. At the time of the original search, the journal reference record was available in PubMed and 'Ovid Medline In-Process \& Other Non-Indexed Citations' but not the version of Ovid Medline searched (Ovid MEDLINE(R) < 1946 to April Week $32,017>$ ). Of the remaining 5 primary references, CINAHL contained 5 but only retrieved 2, EMBASE contained 4 but only retrieved 1, Ovid Medline (1946- 


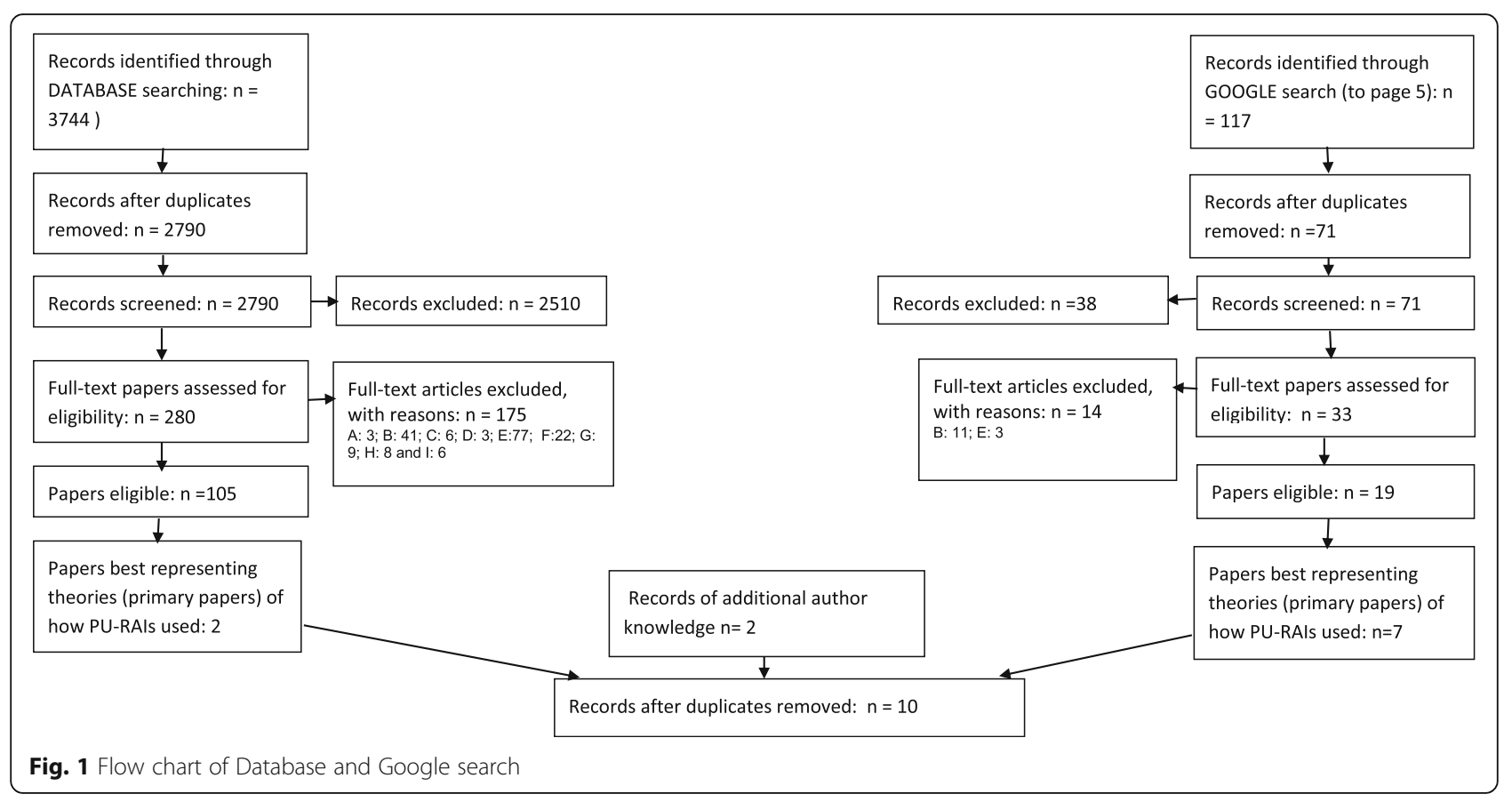

present) contained 4 but only retrieved 1 and The Cochrane Library contained and retrieved 1 . The references found in EMBASE, Medline and The Cochrane Library were duplicates of the references found within CINAHL. Three references were not identified by the academic databases searches despite being available in at least one database $[28,29,32]$ due to using the search limit 'Adult' or 'Aged' which the references had not been indexed for [29, 32]. Also search terms (index terms and free text words) used in the search concept for 'theory and publication types' were not present in some database indexing records $[28,29,32]$.
The optimum search with the lowest NNR of 21.8 was the original Google search (Pressure ulcer risk assessment and Pressure ulcer risk assessment tools). We extended the records screened from five pages (approx. 36 unique records) per Google search to at least 12 pages (approx. 108 unique records) per Google search, in order to retrieve all 10 primary references.

\section{Replicability of Google and Google scholar searches}

The results of the replicability searches in Google where the first five pages were screened by 2 researchers working independently, were similar but not

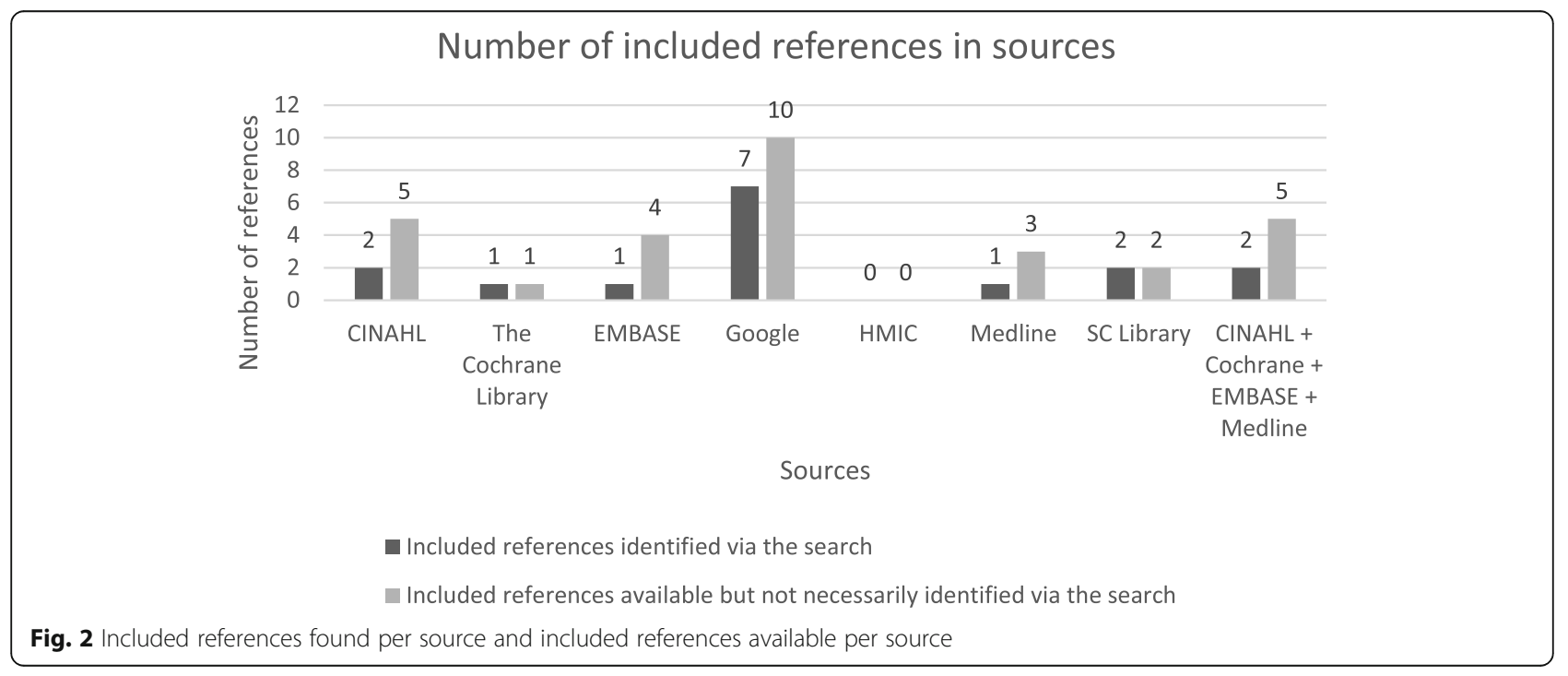




\section{Included references}

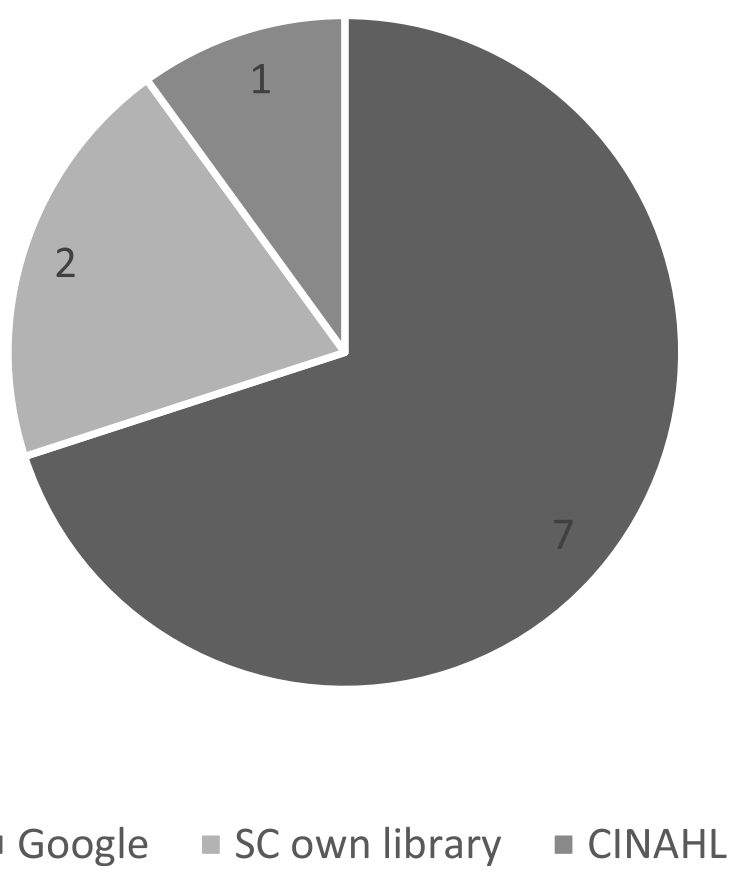

Fig. 3 Minimum sources required to find the 10 included references using our search terms (strategy) identical. For search (i) 'Pressure Ulcer Risk Assessment', 11 results were on the same page for each researcher but in a different order and six references were found by one but not both researchers. For search (ii) 'Pressure Ulcer Risk Assessment Tool', 10 results appeared in a different order for each researcher and 22 results were found by one but not both researchers.

Comparison searches were undertaken to test if Google Scholar performed better than Google Searches for identifying our ten primary papers, and to check replicability. The first five pages for each search $(76$ records in total) were downloaded. The search results from both researchers were exactly the same, indicating strong replicability. However, in both the 'Pressure Ulcer Risk Assessment' and the 'Pressure Ulcer Risk Assessment Tools' search, only one of the ten primary papers was identified [34]. Google Scholar was inferior to Google in this case study for identifying reports identifying programme theories.

\section{Optimising the search}

In light of our experience in this case study we used a combined but adapted approach to help mitigate publication bias that can arise from only using one source for publications, or in the case of Google searching, an 'Internet Research Bubble' [38]. This comprises the existing Google search but extending to page 12 and optimising our academic databases searches by searching only CINAHL, the most relevant database (containing the most included studies (5) with the other databases only containing a subset of these 5). We also optimised the precision of the CINAHL search by searching only

Table 2 Retrieval and Non-retrieval of references across databases and reasons for non-retrieval

\begin{tabular}{|c|c|c|c|c|c|}
\hline \multirow[b]{2}{*}{ Study reference } & \multirow{2}{*}{$\begin{array}{l}\text { Google } \\
\text { Retrieval in Google } \\
\text { (non-retrieval reason) }\end{array}$} & \multicolumn{4}{|l|}{ Academic databases } \\
\hline & & $\begin{array}{l}\text { Retrieval in MEDLINE } \\
\text { non-retrieval reason) }\end{array}$ & $\begin{array}{l}\text { Retrieval in EMBASE } \\
\text { (non-retrieval reason) }\end{array}$ & $\begin{array}{l}\text { Retrieval in CINAHL } \\
\text { (non-retrieval reason) }\end{array}$ & $\begin{array}{l}\text { Retrieval in Cochrane } \\
\text { (non-retrieval reason) }\end{array}$ \\
\hline Bell 2005 & $\checkmark$ & $x$ & $x$ & 3 & $x$ \\
\hline Chapman 2014 & $\checkmark$ & $x$ & $x$ & $x$ & $x$ \\
\hline Guy 2012 & $\checkmark$ & 2,3 & 2,3 & 3 & $x$ \\
\hline Green 2017 & $\checkmark$ & $x$ & $x$ & $x$ & $x$ \\
\hline Fletcher 2017 & $\checkmark$ & $x$ & 2,3 & 3 & $x$ \\
\hline Torra i Bou 2006 & $\checkmark$ & $x$ & $x$ & $x$ & $x$ \\
\hline Moore 2014 & $\checkmark$ & 2 & $x$ & $\checkmark$ & $\checkmark$ \\
\hline Johansen 2014 & 1 & $\checkmark$ & $\checkmark$ & $\checkmark$ & $x$ \\
\hline Kottner 2010 & 1 & 4 & 4 & $x$ & $x$ \\
\hline EPUAP/ NPUAP 2014 & 1 & $x$ & $x$ & $x$ & $x$ \\
\hline
\end{tabular}

$\mathrm{X}$ : the reference was not stored (or indexed) in the database at time of search

Reasons for non-retrieval when database did contain the reference

1: not within first 5 pages of Google search

2: No age limit indexing

3: No terms from theory and publication types search concept

4: In PubMed but not Ovid Medline 1946-present 
in the title field and not the abstract or keywords for the phrases "pressure ulcer" "pressure ulcers" AND "risk assessment". This would identify the same five available primary references from CINAHL with fewer abstracts needing to be screened. To demonstrate this we ran the specific phrase search in CINAHL (for the same time period of our original search). Search results were limited to studies published up to May 2017, when the original CINAHL search was conducted) and 186 papers were identified with an NNR of 18.6.

The Google and CINAHL optimised searches retrieved 402 references with an NNR of 40.2, whereas the original searches retrieved $(2790+71+2) 2863$ references with an NNR 286.3. The researcher would save time in screening 2461 (2863-402) fewer references, and also by not developing complex search strategies and downloading records from four academic databases. If we estimate that it takes on average 2 min to screen each paper with $10 \%$ being screened by a second reviewer this would save approximately $90 \mathrm{~h}$ of researcher time. This illustrates the large difference in efficiency of the optimised search to the original, though we acknowledge it is impossible to develop such an optimised search when you are developing literature search at the start of the project as you do not yet know the included studies the search needs to find. However, the results indicate some search methods worthy of further testing and research for more efficient identification of programme theory.

\section{Discussion}

Frustration in the results of a search of academic databases for identifying sources of programme theory associated with PU-RAI use (where only 2/10 primary papers found) led to analysis of the search strategy, databases and Google search to understand why. When considering the original database searches for this study we found the same results could have been achieved by searching just CINAHL rather than five databases. This is because pressure ulcers studies are usually conducted by nurses, are most likely to be published in nursing journals and indexed in nursing databases. CINAHL is the only nursing database our institution subscribes to so we could only analyse this. Future research could compare and test the performance of searching other nursing databases e.g. EMCARE, BNI to retrieve programme theory related to PUs. It is acknowledged that the most appropriate database will vary for different subject areas and the scope of the programme theory research questions, e.g. those wider in scope may require consideration of a range of subjects or health specialities, requiring searches of a more multi-disciplinary database, or several databases. Our findings indicate that where the research question relates to a specific speciality covered in depth by a subject database, it could be most efficient to focus searches in the subject database. Further research is required to test if this is the case for other specialities and their subject databases.

The results of the database search highlight inconsistent indexing for age of study population preventing identification of all 5 primary references available. To improve the performance of academic databases in future searches it would be better to identify and then remove 'child or adolescent' studies from the search results rather than use the 'adult' filter. The original searches would have also found more primary papers if they had not used a 'theories and publication type' search concept. It could be argued that the original database search could have been improved and avoided these pitfalls if it had been conducted by an expert searcher rather than an applied health researcher who is less aware of the complexity of searching.

The comparison of the combined academic databases searches (NNR: 1395) with a simple key phrases Google search (NNR: 10.1) found substantially improved precision and sensitivity with the Google search. Given that many primary sources of relevance to programme theory scoping reviews are not indexed in academic databases, they cannot not be retrieved via traditional academic searches. While this may be obvious to those with methodological expertise in the field, for those moving from more traditional research paradigms to realist approaches, this is an important consideration for their search strategies. Indeed, searching grey literature and using supplementary search techniques are considered as important, if not more important than searches of academic literature [39-41], as they can provide contextual richness and reduce bias introduced from peerreviewed journal articles. Search engines such as Google provide an accessible route to grey literature as well as academic studies that may have been missed in a database search [38, 42]. They provide links to web-based content and reports that can be particularly useful for identifying programme theories and not available in academic databases. For example, a Google search could uncover a blog post or newspaper article discussing opinions of why an intervention works in one setting but not another. This blog data on ideas and assumptions can inform a programme theory and would not be found via an academic database search.

However, the use of search engines such as Google and Google Scholar is controversial in conventional systematic review search methods. They may provide access to large quantities of academic literature and a wide range of 'grey' literature of relevance to systematic reviews, but they cannot be searched in the reproducible manner required by conventional systematic reviews [39]. Google search results are personalised in order to display and prioritise results and advertisements it 
considers most pertinent to the searcher. The exact algorithms used to personalise searches are unknown but are based on factors such as the geographical location of the PC IP address, previous Google searches, and links clicked by the searcher [22]. Personalised search results can introduce bias into systematic reviews by a 'search bubble effect' where the tailored search results reflect the searcher's interests and preferences rather than an impartial representation of websites on the topic searched [38]. It is possible to reduce the personalisation of Google searches and improve their impartiality by using Verbatim search functions, ensuring you are logged out of Google personal accounts, using a private browser window or incognito (Chrome) $([22,38]$, and by searching for negative as well as positive views of the intervention under study.

Personalisation of searches may be less of a problem for programme theory reviews, where the aim is to search for each different idea/assumption underlying the intervention under study (rather than trying to comprehensively identify every study with relevant data for a conventional systematic review). Furthermore, the task is to identify plausible programme theories, rather than evidence to underpin the validity of those ideas (which occurs in the next stage of a realist review or evaluation). The ranking of results may favour items and websites that are already well known and visited by the researcher, or websites which are geographically close. For programme theory reviews this may be helpful in quickly identifying highly relevant material if the searcher (and the PC they use) has a history of accessing websites and publications on the topic of interest, leading to more relevant items appearing higher up in the ranked Google search results. Unfortunately though, the searcher cannot feel confident they have identified a set of results that is free from bias towards their geographical locations or organisations they are connected with (via Google searches). Search personalisation also remains a problem for developing transparent and replicable results as two searchers using the same search strategy may retrieve different results as noted in our replica Google searches.

Google searches may provide a useful means of informing search terms to be used in academic database searches. Further consideration is needed regarding the impact of cookies, search history and the use various IP addresses in future work. In addition, there is a need to explore and compare alternative search engines to Google such as Bing and DuckDuckGo and different sources of grey literature such as WorldCat, NICE Evidence, OpenGrey in comparison with Google.

Using this case study, we were able to identify an optimum search strategy for programme theories associated with PU-RAI use. Through developing the optimum search strategy, we identified search methods that may help develop more efficient searching for programme theory searches in the future. Searching Google and screening the first 216 (approximately 12 pages) allowed us to find important papers that would otherwise have been missed. If searchers usually screen only the first few pages of Google results, they should consider screening more pages as our case study and guidance for searching grey literature in systematic reviews suggests highly relevant papers are being found in the first 200300 results [43]. The use of an academic database search (incorporating precise key phrases) in the optimised search recognises that searchers would not feel confident relying on a Google-only search. Searching an academic database provided added transparency and replicability to the searches.

While it is not possible to create such an optimised search when first developing searches for either a systematic or programme theory review, the iterative nature of programme theory development lends itself to testing alternative approaches to identifying relevant papers.

$t$ should also be recognised that the terms used in the database search strategies were not directly comparable with the Google search terms, however it is an example of a real-world search done by a non-expert researcher. Furthermore, we only evaluated the databases and Google for the included studies we had already found. Using other sources may have allowed us to identify additional papers for inclusion but we were unable to evaluate this in the present case study.

What remains unclear is whether our example is typical of other programme theory searches and further research is needed to establish if our results are generalisable to other subject areas. Nevertheless, the findings provide a useful insight which could help researchers undertaking searches for programme theory reviews, rather than conventional systematic reviews. We hope the results of this study can be combined with others in the future to build a more robust evidence base for such searches and inform methodological advancement in the field.

\section{Conclusion}

Limitations of database searches for identifying programme theories associated with PU-RAI use led to consideration of other sources and comparison of academic database and Google searching. The findings indicate the importance of including grey literature sources such as Google in this programme theory search, while acknowledging the need for transparency and replicability. The comparison led to the development a combined and optimised search strategy to efficiently capture primary papers incorporating the extension of the Google search to screen the first 12 pages (rather than the first 5 pages) and a precise search of one large academic database most closely aligned with our topic. 
Further research is needed to establish whether these principles are generalisable to other subject areas. This would facilitate further guidance for programme theory searches to enhance practice in the realist field with implications for saving researcher time and therefore resource.

\section{Supplementary information}

Supplementary information accompanies this paper at https://doi.org/10. 1186/s12874-020-01084-X.

Additional file 1.

\section{Abbreviations}

NNR: Number Needed to Read; PU: Pressure Ulcer; RAl: Risk Assessment Instrument

\section{Acknowledgements}

None.

\section{Authors' contributions}

I can confirm that all authors have made substantial contribution to the paper and meet the criteria for authorship as detailed below: SC, contributed to the conception and design of the study, co-ordination the study, data collection (acquisition of data), analysis and interpretation data, drafted and revised the article and final approval of the version to be submitted. JW, contributed to the conception and design of the study, analysis and interpretation of data, drafted and revised the article and final approval of the version to be submitted. JN, contributed to the conception and design of the study, interpretation of data, revising the article and final approval of the version to be submitted. LS, contributed to the conception and design of the study, interpretation of data, revising the article and final approval of the version to be submitted. MT, contributed to the conception and design of the study, interpretation of data, revising the article and final approval of the version to be submitted. JG, contributed to the conception and design of the study, interpretation of data, revising the article and final approval of the version to be submitted.

\section{Funding}

This report is independent research arising from a Post-Doctoral Research Fellowship (Susanne Coleman PDF 2016-09-054), supported by the National Institute for Health Research. The views expressed in this publication are those of the author(s) and not necessarily those of the NHS, the National Institute for Health Research, Health Education England or the Department of Health.

\section{Availability of data and materials}

The datasets during and/or analysed during the current study available from the corresponding author on reasonable request.

\section{Ethics approval and consent to participate}

Not applicable.

\section{Consent for publication}

Not applicable.

\section{Competing interests}

The authors declare that they have no competing interests.

\section{Author details}

${ }^{1}$ Leeds Institute of Clinical Trials Research, University of Leeds, Leeds LS2 9JT, UK. ${ }^{2}$ University Medical Center Heidelberglaan 100, 3584 CX, Utrecht. Internal mail Str. 6.131 PO Box 85500, 3508 Utrecht, GA, Netherlands. ${ }^{3}$ Mixed Methods Research. Hull York Medical School, University of Hull, Cottingham Road, Hull HU6 7RX, UK. ${ }^{4}$ Department of Sociology and Social Policy, University of Leeds, Leeds LS2 9JT, UK.
Received: 29 April 2020 Accepted: 21 July 2020

Published online: 26 August 2020

\section{References}

1. Moore GF, et al. Process evaluation of complex interventions: Medical Research Council guidance. BMJ. 2015;350.

2. Pawson R, Tilley N. Realist Evaluation. Editor: B.C. Office; 2004. http://www. communitymatters.com.au/RE_chapter.pdf.

3. Wong $\mathrm{G}$, et al. RAMESES II reporting standards for realist evaluations. BMC Med. 2016;14.

4. Richards DA, Hallberg IR. Complex Interventions in Health: An Overview of Research Methods. 2015. London: Routledge Taylor and Francis Group.

5. Randell $R$, et al. Integration of robotic surgery into routine practice and impacts on communication, collaboration, and decision making: a realist process evaluation protocol. Implement Sci. 2014;9(1):52.

6. Pawson, R., L. Owen, And G. Wong. The Today Programme's Contribution to Evidence-based Policy 2010. 16(2): p. 211-213.

7. Booth A, Carroll C. Systematic searching for theory to inform systematic reviews: is it feasible? Is it desirable? Health Information \& Libraries Journal. 2015;32(3):220-35.

8. Booth A, Wright J. And B. S., Scoping and searching to support realist approaches. Doing realist research. 2018. London: sage.

9. $\mathrm{AHRQ}$, Methods Guide for Effectiveness and Comparative Effectiveness Reviews. AHRQ Publication No. 10(14)-EHC063-EF. Chapters available at: WWW. effectivehealthcare.ahra.gov. [accessed 27-06-19]. 2014, Rockville, : MD: Agency for Healthcare Research and Quality.

10. Aromataris E and M.Z. (Editors). Joanna Briggs Institute Reviewer's Manual. The Joanna Briggs Institute. Available from https://reviewersmanual. joannabriggs.org/. 2017.

11. EUnetHTA., Guideline: process of information retrieval for systematic reviews and health technology assessments on clinical effectiveness. Ver 1.2. . 2017, European network for Health Technology Assessment.

12. Lefebvre C, et al., Chapter 6: Searching for studies. In: Higgins JPT, Green S (editors). Cochrane Handbook for Systematic Reviews of Interventions Version 5.1.0 [updated March 2011]. The Cochrane Collaboration, 2011. Available from www.cochrane-handbook.org, 2011.

13. Greenhalgh J, et al. Reviewing and selecting outcome measures for use in routine practice. J Eval Clin Pract. 1998;4(4):339-50.

14. Stevinson C, Lawlor DA. Searching multiple databases for systematic reviews: added value or diminishing returns? Complementary Therapies in Medicine. 2004;12(4):228-32.

15. Greenhalgh, J., et al., Functionality and feedback: a realist synthesis of the collation, interpretation and utilisation of patient-reported outcome measures data to improve patient care. Health Service and Delivery Research, 2018.

16. Greenhalgh T, Peacock R. Effectiveness and efficiency of search methods in systematic reviews of complex evidence: audit of primary sources. BMJ. 2005;331(7524):1064-5.

17. Booth, A., Acknowledging a "Dual Heritage" for Qualitative Evidence Synthesis: Harnessing the Qualitative Research and Systematic Review Research Traditions. 2013, Sheffield: PhD thesis, University of Sheffield.

18. Booth A, Papaioannou D, Sutton AJ. Systematic Approaches to a Successful Literature Review. 2011. London: Sage.

19. Papaioannou D, et al., Literature searching for social science systematic reviews: consideration of a range of search techniques. Health Information \& Libraries Journal, 2010. Jun;27(2): p. 114-22.

20. Briscoe S. Web searching for systematic reviews: a case study of reporting standards in the UK health technology assessment programme. BMC research notes. 2015:8:153.

21. Levay $P$, et al. Identifying evidence for public health guidance: a comparison of citation searching with web of science and Google scholar. Res Synth Methods. 2016;7(1):34-45.

22. Blakeman, K., Top Search Tips http://www.rba.co.uk/search/TopSearchTips.pdf accessed 27-06-19. RBA information, 2018.

23. Greenhalgh, T., et al., Protocol-the RAMESES // study: developing guidance and reporting standards for realist evaluation. BMJ Open, 2015. 5(8).

24. Moore ZE, Cowman S. Risk assessment tools for the prevention of pressure ulcers. Cochrane Database Syst Rev. 2014;2:CD006471.

25. Bethel A and R. M, Search summary table for systematic reviews (SRs). In: Filtering the information overload for better decisions. , in Abstracts of the 23rd Cochrane Colloquium. 2015, John Wiley \& Sons: Vienna, Austria. 
26. Whiting $P$, et al. Systematic reviews of test accuracy should search a range of databases to identify primary studies. J Clin Epidemiol. 2008;61(4):357-64.

27. Levay P and C. J, Systematic Searching 2019, London: Facet publishing.

28. Bell J. Are pressure ulcer grading and risk assessment tools useful. Wounds UK. 2005;1 (2):62-9.

29. Guy H. Pressure ulcer risk assessment. Nurs Times. 2012;103(15):38-40.

30. Chapman, S., Nurses underpressure: do risk assessment tools help prevent pressure ulcers https://evidentlycochrane.wordpress.com/2014/02/14/nursesunder-pressure-do-risk-assessment-tools-help-prevent-pressure-ulcers/. Evidently Cochrane, 2014

31. Green, H., Experience and perceptions using the Waterlow pressure ulcer risk assessment tool: a community care perspective, in Wounds Uk. 2016: Harrogate.

32. Fletcher J. An overview of pressure ulcer risk assessment tools. Wounds UK. 2017:13(1):18-26.

33. Torra i Bou, J.E., et al., Risk assessment scales for predicting the risk of developing pressure ulcers, in Science and Practice of Pressure Ulcer Management.

34. Moore Z, Cowman S. Risk assessment tools for the prevention of pressure ulcers. Cochrane Database of Sysematic Reviews. 2014;2.

35. E. Johansen BsC, M., RN, et al., Pressure ulcer risk assessment and prevention: What difference does a risk scale make? A comparison between Norway and Ireland. 2014. 23(7): p. 369-378.

36. Kottner J, Balzer K. Do pressure ulcer risk assessment scales improve clinical practice. J Multidiscip Healthc. 2010;3:103-11.

37. NPUAP/EPUAP/PPPIA, Risk factors and risk assessment chapter.

38. Curkovic M, Kosec A. Bubble effect: including internet search engines in systematic reviews introduces selection bias and impedes scientific reproducibility. BMC Med Res Methodol. 2018;18(1):130.

39. Adams J, et al. Searching and synthesising 'grey literature' and 'grey information' in public health: critical reflections on three case studies. Systematic Reviews. 2016;5(1):164.

40. Cooper C, et al. Supplementary search methods were more effective and offered better value than bibliographic database searching: a case study from public health and environmental enhancement. Res Synth Methods. 2018;9(2):195-223.

41. Wright JM, Cottrell DJ, Mir G. Searching for religion and mental health studies required health, social science, and grey literature databases. J Clin Epidemiol. 2014;67(7):800-10.

42. Stansfield C, Dickson K, Bangpan M. Exploring issues in the conduct of website searching and other online sources for systematic reviews: how can we be systematic? Syst Rev. 2016;5(1):191.

43. Haddaway NR, et al., The role of Google Scholar in evidence reviews and its applicability to grey literature searching. PloS one, 2015. 17(10): p. 9): e0138237.

\section{Publisher's Note}

Springer Nature remains neutral with regard to jurisdictional claims in published maps and institutional affiliations.

Ready to submit your research? Choose BMC and benefit from:

- fast, convenient online submission

- thorough peer review by experienced researchers in your field

- rapid publication on acceptance

- support for research data, including large and complex data types

- gold Open Access which fosters wider collaboration and increased citations

- maximum visibility for your research: over $100 \mathrm{M}$ website views per year

At $\mathrm{BMC}$, research is always in progress.

Learn more biomedcentral.com/submissions 